\title{
1 Ergodic patterns of cell state transitions underlie the reproducibility of
}

\section{2 embryonic development}

3 Miriam Genuth ${ }^{1, \dagger}$, Yasuhiro Kojima ${ }^{1,2,3, \dagger}$, Dörthe Jülich ${ }^{1}$, Hisanori Kiryu ${ }^{3}$ and Scott A.

4 Holley $^{1 *}$

$5 \quad{ }^{1}$ Department of Molecular, Cellular and Developmental Biology, Yale University, New Haven, CT,

6 USA. ${ }^{2}$ Division of Systems Biology, Graduate School of Medicine, Nagoya University, Nagoya,

7 4668550, Japan. ${ }^{3}$ Department of Computational Biology and Medical Sciences, Graduate School

8 of Frontier Sciences, The University of Tokyo, Kashiwa, Chiba, Japan.

9 The authors contributed equally to this work.

10 *Corresponding Author: scott.holley@yale.edu 


\section{Abstract}

2 The reproducibility of embryonic development is a remarkable feat of biological organization, but

3 the underlying mechanisms are poorly understood. Clearly, gene regulatory networks are central

4 to the orderly progression of development, but noisy molecular and cellular processes should

5 reduce reproducibility. Here, we identify ergodicity, a type of dynamical stability, as underlying the

6 reproducibility of development. In ergodic systems, a single timepoint measurement equals a time

7 average. Focusing on the zebrafish tailbud, we define gene expression and cell motion states

8 using a parallel statistical analyses of single cell RNA sequencing data and in vivo timelapse cell

9 tracking data and a change point detection algorithm. Strikingly, the cell motion state transitions

10 in each embryo exhibit the same patterns for both a single timepoint and a 2-3 hour time average.

11 Both the cell motion and gene expression cell states exhibit balanced influx and outflux rates

12 reflecting a spatiotemporal stability. Stated simply, these data indicate the pattern of changes in

13 the tailbud doesn't change. This ergodic pattern of cell state transitions may represent an

14 emergent meta-state that links gene networks to the reproducible progression of embryogenesis. 


\section{Introduction}

2 Aristotle first noted the astonishing reproducibility of embryogenesis in "Historia Animalium,"

3 where he observed, "Generation from the egg occurs in an identical manner in all birds". At the

4 cellular level, development entails a reproducible series of cell state transitions representing

5 changes in gene expression state, physical state and cell fate. These processes can be noisy, for example, cell migration can be either ordered or disordered, and such disorder is part of normal orderly development. We now appreciate that gene networks control cell state transitions, but these networks are comprised of stochastic molecular processes. How biological order emerges from stochastic molecular events was the subject of Erwin Schrödinger's "What is Life? ”. Despite the remarkable progress in the field of developmental biology in recent decades, there is still a gap in our understanding of the organizing processes that lie between genes and the reproducible dynamics of developing embryos.

One framework for analyzing the reproducibility of embryonic development is that of ergodicity.

An ergodic system is one in which the average behavior of all objects at a single timepoint equals the average behavior of a random sample of objects over a longer time interval. For example, measurement of all gas molecules within a chamber at a single time point yields the same result

17 as the average of a random sample of gas molecules over the entire experimental time interval.

The ergodic hypothesis lacked a mathematical foundation until the development of the ergodic theorem in 1932 . In the field of ergodic theory, the single timepoint average is typically referred to as the "phase average". Ergodicity is a mathematical ideal and real systems are not truly ergodic. Embryonic development is by definition not ergodic since the embryo changes as it develops, but it is possible for ergodicity to exist over a short period of time. Ergodicity is implicitly assumed in many biological experiments, yet it is rarely demonstrated. For example, ergodicity 
1 Here, we address the mechanism of the reproducibility of embryonic development by performing

2 an analysis of ergodicity of the pattern of cell state transitions in the zebrafish tailbud. The

3 vertebrate tailbud is a dynamic structure that supports body elongation (Fig. 1a, left panel). Cells

4 in the tailbud undergo multiple transitions in gene expression and migratory behavior during their

5 differentiation. The dorsal-medial tailbud (DM) contains a pool of sox2/brachyury expressing

6 neuromesodermal progenitors (NMPs) that contribute to both the spinal cord (Fig. 1A, yellow) and

7 the presomitic mesoderm (PSM). In the zebrafish, cells in the DM migrate towards the posterior

8 in a processive orderly fashion (Fig. 1A, cyan). At the tip of the tailbud, mesodermally fated cells

9 downregulate sox2, upregulate mesodermal genes such as $t b x 16$, and undergo EMT to migrate

10 ventrally into the progenitor zone (PZ)(Fig. 1A, magenta). Cell movements in the PZ are more disorderly than the DM. Ultimately cells leave the PZ, reduce their cell motion and assimilate into

12 the left and right PSM (Fig. 1A, green). Cells in the PSM downregulate tbx16 and turn on tbx6.

13 Cell velocity in the anterior PSM declines further as the tissue solidifies. The transition from orderly to disorderly motion from the DM to $P Z$ is necessary for proper body elongation . Excessively disordered motion in the DM (obtained by inhibition of BMP or FGF signaling) impairs the flow of cells through the tailbud leading to a short body axis. Excessively ordered motion in the PZ (induced by moderate Wnt inhibition) produces prolonged anisotropic fluxes, unequal allotment of cells to the left or right PSM, and a bent body axis. Thus, understanding robustness and reproducibility of vertebrate body elongation requires understanding the nature of these tailbud cell state transitions.

21 In this study of ergodicity and the reproducibility of development, we first objectively define the 22 trajectory of cell states in the zebrafish tailbud during body elongation. We define gene expression 23 state using single cell RNA sequencing (scRNAseq) and a change point detection algorithm. We 24 validate this method by comparing wild type and embryos with reduced Wnt, Fgf and Bmp 25 signaling, and verify quantitative differences in cell states by multicolor fluorescent in situ 
1 hybridization. Next, we identify cell motion states by analyzing cell tracking data using the same

2 statistical analysis as used for scRNAseq. We then perform an analysis of ergodicity of the pattern

3 of cell motion states as these datasets allow direct comparison of a single timepoint with a time

4 average. We find that the ergodicity is achieved via a balanced flux between cell motion states in

5 each embryo. Since it is not possible to directly track gene expression states over time in

6 scRNAseq data, we estimate the flux between the gene expression states from RNA velocity and

7 confirmed balanced flux in wild-type embryos. These consistently balanced fluxes for both cell

8 motion and gene expression states suggest that the ergodicity is an emergent order which can

9 explain the reproducibility and robustness of embryonic development.

\section{Results}

\section{Gene expression states}

12 We performed scRNAseq on dissected tails from 10-12 somite stage zebrafish embryos (Fig. 1A).

13 We used wild-type embryos and embryos subject to treatments known to alter tailbud cell migration, specifically inhibition of FGF, BMP, or Wnt signaling . For each treatment, we prepared four biological replicates each consisting of 10 to 12 tailbuds and resulting in $30,000-35,000$ single cell profiles. In a UMAP dimension reduction plot of wild type, the neuronal and paraxial mesoderm form one large cluster with more differentiated cells at each end and common progenitors (cyan) in the middle (Fig. 1B, arrow, and Fig. S1). Wild-type and experimental samples consist of the same cell transcription profiles (Fig. 1D). This result is consistent with previous scRNAseq analysis of zebrafish embryos indicating that perturbation of cell signaling

21 does not create novel cell transcription profiles.

22 To enable direct quantitative comparisons between experimental conditions, we pooled the data

23 from all wild-type and experimental replicates and created one unified pseudotime to define a 24 single standard for classifying cells. Specifically, the cells in the main cluster were aligned along 
1 a neuronal-mesodermal axis from sox3 expressing neuronal cells to mespaa expressing anterior

2 PSM cells (Fig. 1B, arrow). This approach avoids the requirement to define the NMP population

3 a priori. Instead, NMPs will be located in the middle of the pseudotime sequence and

4 differentiation will proceed towards both ends, i.e. neuronal to the left and mesodermal to the right

5 (Fig. 1C). Marker genes for neuronal and mesodermal development map with respect to

6 pseudotime in the correct developmental sequence indicating that the procedure was successful.

7 To objectively define gene expression states, we extracted the wild-type data, and then utilized a change point detection algorithm to divide pseudotime into a series of distinct states. The change point algorithm identified five transition points (Fig. S2). These transition points (vertical lines in Fig. 1C) divide the pseudotime sequence into six states that generally agree with those predicted previously from marker gene expression. These transition points were mapped to the full pseudotime sequence, and we calculated the relative abundance of each state in wild type, Wnt inhibited embryos, Fgf inhibited embryos and Bmp inhibited embryos (Fig. 1D).

To determine whether this analysis of scRNAseq data accurately quantifies changes in cell state, we mapped the transcriptional states back onto the embryo and measured their abundance using simultaneous multicolor fluorescent in situ hybridization for marker genes for the first five states

17 (Fig. 2A). Sox2 single positive cells localize in the neural tube (state 1). Sox2 and brachyury positive NMPs (state 2) occupy the DM. Nascent mesodermal progenitors (state 3) expressing brachyury and tbx16 are located immediately ventral to the DM in the medial PZ. Mesodermal progenitors in the $\mathrm{PZ}$ (state 4) are tbx16 single positive cells located in the ventral and lateral tailbud. The PSM (state 5) is anterior to the transition from tbx16 to tbx6 expression. of neuronal and PZ states. First, the scRNAseq predicts that Wnt inhibited embryos would have more neuronal cells (Fig. 1E). This is consistent with reports that elimination of Wnt signaling 
1 of embryos have an abnormal cap of neuronal tissue covering the embryos' posterior, confirming

2 the scRNAseq results (Fig. S3).

3 A second prediction of the scRNAseq analysis is that the PZ is smaller in BMP and Wnt inhibited embryos but not in embryos subject to FGF inhibition. To test this prediction, we performed fluorescent in situ hybridization for a PZ marker, tbx16, and a PSM marker, tbx6 (Fig 2B). In wildtype, BMP and FGF inhibited embryos, the $t b \times 16$ and $t b x 6$ signal was measured along the anterior-posterior axis of the embryo for both the left and right sides (Fig. 2C). The PZ/PSM transition was set to the value derived from the scRNAseq analysis ( $20 \%$ of the maximum value of $t b \times 6)$ and then the PZ length was normalized to the total tailbud length. Consistent with the scRNAseq analysis, BMP but not FGF inhibited embryos exhibited a decrease in PZ length (Fig. PZ and PSM were quantified. As predicted, Wnt inhibited embryos have a smaller PZ (Fig. 2E). Thus, this approach to analyzing scRNAseq data accurately identifies cell states that can be quantitatively mapped back onto the embryo.

\section{Cell motion states}

We hypothesized that the same computational techniques used to classify gene expression states could be applied to cell motion data to objectively define the cell motion states (Fig. 3A). For this purpose, we used tracking data from confocal timelapse imaging of cells in the DM through PSM collected over 1-3 hours in wild-type embryos and embryos subject to signaling perturbations . As with the gene expression analysis, the cell motion statistics for each cell track were used to order the tracks in pseudotime, and the state transitions were defined using the change point detection algorithm. The cell states were color coded and spatially mapped back onto the embryo using the original cell track position. Initially, we chose not to use cell position as a pseudotime input both

24 to facilitate pooling of data from multiple embryos together and to make the analysis analogous

25 to that of the scRNAseq data which had all spatial information removed by cell dissociation. This 
1 procedure is successful solely using the statistics for cell velocity, average neighborhood cell

2 speed within 20 micron radius of each cell, acceleration, and displacement over 6 and 15 minutes

3 (Fig. 3B). The change point detection algorithm classifies the cells into four cell motion states (Fig.

4 S4). These states are roughly segregated in space and their sequence matches the known

5 developmental trajectory. Thus, cell migration states can be considered analogous to gene

6 expression states.

$7 \quad$ An ergodic pattern of cell motion states

8 The cell tracking data includes cell position, and we postulated that utilizing this information would

9 improve the cell state segmentation. We therefore created a cell state map for each embryo using

10 cell position and cell track displacement as inputs for pseudotime assembly (Fig. 3C, 3D and S5).

11 These pseudotime sequences were then segmented based on the aforementioned cell motion

12 statistics. This approach cleanly segmented the embryo into four cell states. As each embryo

13 contains tens of thousands of data points, we plotted only a sample of the data from either a single

14 time point, i.e. a phase average (Fig. 3C), or an identically sized selection randomly chosen from

15 all time points, i.e. a time average (Fig. 3D). The distribution of states is extremely similar in both

16 plots which is indicative of an ergodic system. The stability of the cell state pattern is evident in a

17 movie generated using the average of each timepoint of our longest wild-type dataset (Movie S1).

18 To obtain further evidence of ergodicity, we measured the cell abundance in each state over time as well as the influx and outflux from these states. The expectation is that the size of these domains would remain constant, and the fluxes would balance. We focused on the two states in

21 the middle of the sequence, the PZ and posterior PSM, since we have both their complete influx

22 and outflux data. Interestingly, the abundance and dynamics of these states can vary substantially

23 from embryo to embryo and treatment to treatment, but the fluxes are balanced in each embryo 
1 (Fig. 3E and F). The balanced fluxes would help maintain the ergodic pattern of cell state

2 transitions.

3 Given the stability of the migration state transitions, we wondered whether the transitions between

4 gene expression states were also ergodic. Since RNA sequencing is an endpoint assay that does

5 not readily lend itself to the calculation of time averages, we utilized RNA velocity, which considers

6 the relative amount of intron and exon RNA for each gene, to estimate the flux between states .

7 As expected, the overall RNA velocity is directed down the path of mesoderm differentiation (Fig.

$84 \mathrm{~A}$ and S6). Flux was calculated as the proportion of cells that transitioned to a different state

9 (Fig. 4B and S7). For wild-type, the influx generally matches the outflux suggesting that the size of these domains is stable and that the patterns of cell state transitions may be ergodic. However

11 unlike in the cell motion states, the balance between influx and outflux can be altered by

12 perturbation of cell signaling.

13 A batch of sibling embryos at roughly, but not exactly the same stage in development, produce 14 very reproducible patterns of gene expression (Fig. 4C). In the tailbud, the consistency of this 15 pattern is remarkable given the dynamics of cell motion that are driving elongation of the body 16 axis. However, if the pattern of state transitions in cell motion is ergodic, it is not surprising that 17 the gene expression patterns are also likely ergodic.

\section{Discussion}

19 The ergodic pattern of cell state transitions may represent an emergent level of biological order

20 that mediates gene network actuation of the stereotypical progression of embryogenesis. Our

21 parallel analysis of gene expression and cell migration states using dimensional reduction and a

22 change point detection algorithm demonstrates that these cell state transitions can be objectively

23 defined and mapped back onto the embryo. While any time series dataset is well suited for an 
1 analysis of ergodicity, starting with cell state identification enables detection of ergodicity in

2 complex datasets and reveals higher order ergodic patterns.

3 Ergodicity normally refers to a single stable state in which a dynamical system resides for a given

4 amount of time. The length of time that a system remains in this state is referred to as the sojourn

5 time. In this study, ergodicity refers to a pattern of successive cell states that remains stable over

6 a period of 2-3 hours. Thus counterintuitively, this ergodicity does not mean that the tailbud

7 doesn't change, but that the pattern of changes doesn't change. The ergodic pattern of cell state transitions could be thought of as a "meta-state".

This biological ergodicity is a dynamic order that arises from the genome and the biochemical and physical interactions among cells in space and time. This ergodicity is dependent upon the length of the time interval being studied. If one were to combine data from a gastrula with data from an embryo during body elongation, then there would likely be no ergodicity. Thus, there is a sojourn time for a given pattern of cell state transitions that will scale with the developmental process under study. A given ergodic pattern may exhibit a sojourn time of hours in the case of the zebrafish tailbud or years in the case of adult homeostatic tissues. During development, the embryo may transition from one ergodic pattern to another as it develops until it reaches the relatively long sojourn time of homeostatic tissues in the mature organism.

An innovation of this study is the finding that cell position and cell motion statistics are sufficient to identify cell states via dimensional reduction and subsequent segmentation into cell states using the change point detection algorithm. These dimension reduction techniques, developed to analyze scRNAseq data, can be applied to other complex datasets along with the change point detection algorithm to identify underlying patterns. The methodology presented here provides a way to assay the validity of the assumption of ergodicity, to identify experimental conditions in which ergodicity is lost, and to measure the time intervals over which ergodicity is maintained in 
1 breakdown of homeostasis due to mutation, aging or disease could be quantified via an analysis

2 of ergodicity.

3 C. elegans embryos are famous for their invariant cell lineages in that one embryo develops in

4 exactly the same manner as any other C. elegans embryo . Vertebrate embryos do not display

5 these invariant cell lineages, but fate mapping demonstrates that subpopulations of cells reproducibly give rise to specific tissues in every embryo of a given species. Thus, while $C$. elegans development is precisely reproducible down to the cellular level, vertebrate embryonic development is reproducible down to the level of ensembles of cells. The question is how is this reproducibility achieved in vertebrate embryos? Some of the reproducibility of development is due development. For example, neuromesodermal progenitors transition to mesodermal progenitors, then to presomitic mesoderm and then to somites. It follows that in vertebrate embryos, these gene regulatory networks operate at the level of ensembles of cells as reflected in the concepts of developmental regulation and community effect. This study finds that the pattern of these transitory cell states is ergodic and therefore dynamically stable over time. The fact that the pattern of cell state transitions doesn't change indicates that the rates of change are stable. The

17 absolute cell state influxes and outfluxes vary significantly between embryos but are balanced in each embryo. Thus, ergodicity exists at a higher level, the derivative, and may represent an emergent systems-level order linking gene regulatory networks with the general reproducibility of embryonic development.

\section{Acknowledgements}

23 We thank Abdel-Rahman Hassan for thoughtful discussions, Sarah Smith, A-R Hassan, Abby

24 Kindberg and Holger Knaut for comments on the manuscript, Guilin Wang, Mei Zhong, the Yale 
1 Keck Biotechnology Resource Laboratory and the Yale Stem Cell Genomics Core Facility for help

2 with the scRNAseq.

\section{$3 \quad$ Funding}

$4 \quad$ National Institute of Health grant 1F32GM137502-01 (MAG)

5 National Institute of Health grant R01GM129149 (SAH)

\section{Author Contributions}

7 MAG performed wet lab experiments, data analysis, data interpretation and wrote the manuscript,

8 YK led the data analysis, performed data interpretation and wrote the manuscript, DJ contributed

9 to the wet lab experiments, HK interpreted data and supervised data analysis, and SAH conceived

10 of and supervised the project, interpreted data and wrote the manuscript.

11 Declaration of Interests

12 The authors declare no competing interests.

\section{Materials and Methods}

\section{Data and code availability}

The scRNAseq data has been archived at NCBI GEO (accession no: GSE173894).

\section{Zebrafish methods}

17 Tüpfel-longfin zebrafish were raised according to standard protocols approved by the

18 Institutional Animal Care and Use Committee. Experiments were performed before sex determination in zebrafish. FGF, BMP, and Wnt signaling perturbations were performed using

20 protocols previously developed to modulate cell migration. Specifically, starting at the 6-somite

21 stage embryos were incubated in $50 \mathrm{mM}$ of SU5402 or $40 \mathrm{mM}$ of DMH1 for two hours to inhibit

22 FGF or BMP signaling, respectively. Wnt signaling was inhibited by injecting notum-1 mRNA at 
1 a concentration of $150 \mathrm{ng} / \mathrm{mL}$ into embryos at the single cell stage and then incubating them

2 until the 10-somite stage. This treatment yields a phenotypic spectrum, and embryos with

3 nascent body elongation defects were chosen for further experiments.

\section{$4 \quad$ Tailbud Dissections and scRNA sequencing}

5 Embryos were incubated until the 10-12 somite stage and then dissected in ice cold Hank's

6 Balanced Salt Solution. The tail was collected by cutting immediately posterior to the last formed

7 somite. Groups of tails consisting of ten tails for wild-type, FGF, or BMP inhibition or twelve tails

8 for Wnt inhibition were pooled together. Cells were dissociated by incubation in $20 \mathrm{U} / \mathrm{mL}$ papain

9 solution (Worthing Biochemical) for 15 minutes at $29^{\circ} \mathrm{C}$ with gentle agitation. Halfway through

10 the incubation the solution was triturated ten times with a P200 pipette. Cells were spun down at

$11300 \mathrm{~g}$ for five minutes and then resuspended in $40 \mathrm{~mL}$ of cold HBSS. Cell concentration and

12 viability were checked with a hemocytometer and the volume of the solution was adjusted if

13 required.

Construction of 10X Genomic Single Cell 3' RNA-Seq libraries (Version 3) and sequencing with an Illumina HiSeq4000.

GEM Generation and Barcoding. Single cell suspension in RT Master Mix was loaded on the Single Cell A Chip and partition with a pool of about 750,000 barcoded gel beads to form nanoliterscale Gel Beads-In-Emulsions (GEMs). Each gel bead has primers containing (i) an Illumina R1 sequence (read 1 sequencing primer), (ii) a 16 nt 10x Barcode, (iii) a 10 nt Unique Molecular Identifier (UMI), and (iv) a poly-dT primer sequence. Upon dissolution of the Gel Beads in a GEM,

21 the primers are released and mixed with cell lysate and Master Mix. Incubation of the GEMs then 22 produces barcoded, full-length cDNA from poly-adenylated mRNA. 
1 Full-length, barcoded cDNA was then amplified by PCR to generate sufficient mass for library

2 construction. Enzymatic fragmentation and size selection were used to optimize the cDNA

3 amplicon size prior to library construction. R1 (read 1 primer sequence) were added to the

4 molecules during GEM incubation. P5, P7, a sample index, and R2 (read 2 primer sequence)

5 were added during library construction via End Repair, A-tailing, Adaptor Ligation, and PCR. The

6 final libraries contain the P5 and P7 primers used in Illumina bridge amplification.

7 Sequencing libraries. The Single Cell 3' Protocol produces Illumina-ready sequencing libraries. A

8 Single Cell 3' Library comprises standard Illumina paired-end constructs which begin and end

9 with P5 and P7. The Single Cell 3' 16 bp 10x Barcode and 10 bp UMI are encoded in Read 1,

10 while Read 2 is used to sequence the cDNA fragment. Sequencing a Single Cell 3' Library

11 produces a standard Illumina BCL data output folder. The BCL data includes the paired-end Read

121 (containing the 16 bp 10x Barcode and 10 bp UMI) and Read 2 and the sample index in the i7

13 index read.

\section{Preprocessing of scRNA sequencing data}

15 We aligned the scRNA-seq data to Grcz11 and demultiplexed using Cell Ranger (10X

16 Genomics). After the generation of expression matrices for each sample, we utilized Seurat v3

17 for preprocessing and clustering of scRNA-seq data. First, we excluded cells with an ectopic

18 number of genes or exceeding a specified percentage of mitochondrial genes (Table S1) based

19 on visual inspection for the distribution of these statistics. After the filtering genes, we conducted

20 integration following Seurat's SCTransform integration.

21 We applied principal components analysis and embedded the 30-dimensional PCA coordinates

22 into 2 dimensional UAMP. We clustered cells by Seurat function "FindClusters" with a resolution

23 parameter of 0.5 . 
1 To recover cell state dynamics encoded in the gene expression data, we ordered a subset of

2 scRNA-seq cells which belong to the axis from ADM to PSM so that its ordering recapitulates the

3 developmental trajectory during body elongation. In particular, we embedded the z-scores of 30

4 dimensional PCA coordinates of cells belonging to specified clusters (Sox3+, Sox2+, DM, PZ,

5 pPSM and aPSM) into one dimensional UMAP coordinates. Here, we expected that the most

6 variable axis within gene expression space during this process would be the developmental

7 trajectory. For UMAP embedding, we used the "umap-learn" package in Python and set

8 "n_neighbors" as 400 and "min_dist" as 0.1.

\section{9}

\section{Segmentation of scRNA-seq pseudotime}

We segmented the pseudotime trajectory of scRNA-seq into several segments within which each cell $c$ has similar z-scores of 30-dimentional PCA coordinate $x_{c}$ in order to dissect the dynamics along the progression of cell state transitions during zebrafish body elongation. We utilized a Bayesian algorithm of change detection to find break points of segments $b_{k}(k=1, \ldots, K)$ which minimize the total error from the mean of profile of the segment $\sum_{k} E_{k}$ where $E_{k}=\sum_{c \in C_{k}} V_{c}, V_{c}=$ ||$x_{c}-\mu_{k}||^{2}, \mu_{k}=\frac{1}{\left|C_{k}\right|} \sum_{c \in C_{k}} x_{c}, \quad C_{k}=\left\{c \mid b_{k-1}<\tau_{c}<b_{k}\right\}$ and $\tau_{c}$ is the discretized rank of the estimated pseudo time. We discretized the pseudotime rank into 30 bins for computational efficiency. We determined $\mathrm{K}$ as 5 scRNA-seq data using the elbow method which chose a saturation point along the group variation curve as function of the number of clusters.

\section{Flux analysis based on RNA velocity}

We recovered cell state dynamics behind scRNA-seq data using scVelo which estimates the velocity of RNA for each single cell Using a computed velocity $v_{c}$ of cell $c$ with its single cell transcriptome $x_{c}$, we calculated the predicted transcriptome after a micro duration $\delta$ as $x_{c}^{\prime}=x_{c}+$ $\delta v_{c}$. We set $\delta$ so that $3 \%$ of transcriptome $x_{c}$ changed during $\delta$. We conducted PCA analysis on a concatenated expression matrix of current and $\delta$-elapsed transcriptome and used the 10- 
1 dimensional PCA coordinates of cell $c$ at current and $\delta$-elapsed time points, which we denoted as

$2 \quad z_{c}$ and $z_{c}^{\prime}$ We estimated the segment $b^{\prime}{ }_{c}$ which cell $c$ after $\delta$ is belonging to as the current

3 segment $b_{c^{\prime}}$ of the cell $c^{\prime}$ whose PCA coordinates $z_{c^{\prime}}$ are the nearest to the predicted $\delta$ -

4 elapsed PCA coordinates $z_{c}^{\prime}$. We quantified the transition $F_{k, k^{\prime}}^{\mathrm{RNA}}$ from segment $k$ to segment $k^{\prime}$

5 as the count of cells whose current and $\delta$-elapsed segments are $k$ and $k^{\prime}$ respectively. The

6 normalization is done for the total number of cells within the segment. We also defined the influx

7 rate and outflux rate of segment $k$ as the normalized summation of transition from any segments

8 to $k$ and from $k$ to any segments and defined them as $I_{k}^{\mathrm{RNA}}=\sum_{k^{\prime}} \frac{F_{k^{\prime}, k}^{\mathrm{RNA}}}{N_{k}^{\mathrm{RNA}}}$ and $O_{k}^{\mathrm{RNA}}=\sum_{k^{\prime}} \frac{F_{k, k^{\prime}}^{\mathrm{RNA}}}{N_{k}^{\mathrm{RNA}}}$

9 respectively, where $N_{k}^{R N A}$ is number of cells in segment $k$.

10 Estimation and segmentation of cell movement pseudotime

11 We recovered the pseudo dynamics of the cell movement properties by pseudotime estimation

12 followed by variance minimization segmentation. For pseudotime estimation without positional

13 information, we pooled together the embryos and calculated 1-dimentional UMAP embeddings of

14 each cell from the z-scores of its speed, acceleration, magnitude of neighborhood velocity, and

15 displacement distance for 6 minutes and 15 minutes. The magnitude of neighbor velocity is

16 defined as ||$\sum_{c \in N(c)} v_{c} \|$ where $N(c)=\left\{c^{\prime}|| \mid p_{c}-p_{c^{\prime}} \|<20 \mu m\right\}$ where $p_{c}$ and $v_{c}$ are position

17 and velocity of cell $c$ and $\|$.$\| is the Euclidean norm. For the pseudotime estimation with positional$

18 information each embryo was processed separately. We embedded the z-scores of 3D spatial

19 position and 3D displacement during 15 minutes into one dimensional order using UMAP. For

20 UMAP embedding, we used the "umap-learn" package in Python and set " $n$ neighbors" as 100

21 and "min_dist" as 0.1 . We segmented both types of cell movement pseudotimes using the same

22 methodology for the segmentation of scRNA-seq pseudotime, except that the properties $x_{c}$ for

23 minimizing within-group variation are the z-scores of the speed, acceleration, magnitude of

24 neighborhood velocity, and displacement distance for 6 minutes and 15 minutes. We specified 
1 the number of change points $K$ as 3 using the elbow method which chose a saturation point of

2 along the group variation curve as function of the number of clusters.

3 Flux analysis between cell movement segments

4 We quantified the flux between segments in cell movement data utilizing cell tracking information.

5 We counted the cells which stay at segment $k$ at a time point $t$ and transit to segment $k^{\prime}$ at the

6 subsequent time point $t+1$. In the same way as the previous section, we defined influx rate and

7 outflux rate of segment $k$ as the normalized summation of transition from any segments to $k$ and

8 that from $k$ to any segments and defined them as $I_{t, k}^{\text {mov }}=\sum_{k}{ }^{\prime} \frac{F_{t, k^{\prime}, k}^{\text {mov }}}{N_{t, k}^{\text {mov }}}$ and $O_{t, k}^{\text {mov }}=\sum_{k^{\prime}} \frac{F_{t, k, k^{\prime}}^{\text {mov }}}{N_{t, k}^{\text {mov }}}$

9 respectively, where $N_{t, k}^{\text {mov }}$ is the number of cells in segment $k$ at time $t$.

\section{Multicolor fluorescent in situ hybridization}

11 Probes for sox2, brachyury, tbx16, and $t b x 6$ were purchased from Molecular Instruments. The

12 hairpins and colors are listed in the table below. Staining of 10-12 somite embryos was performed

13 using their recommended protocol with a few modifications. Specifically, batches of 15 embryos

14 were stained simultaneously. The tbx6 probe was diluted 1:10 to avoid excessive bleed through

15 into the sox2 channel. DAPI was added to the amplification mixture. After staining embryos were

16 taken through a series of $25 \% / 50 \% / 75 \%$ glycerol in PBS. The posterior half of the embryo was

17 isolated and mounted dorsal side up in $75 \%$ glycerol. Embryos were imaged with a Zeiss LSM

18880 Airyscan Confocal using a 20x objective.

\begin{tabular}{|l|l|l|}
\hline Gene & Hairpin & Dye \\
\hline Sox2 & B1 & Alexa Fluor 546 \\
\hline Tbx16 & B2 & Alexa Fluor 647 \\
\hline Brachyury & B3 & Alexa Fluor 488 \\
\hline Tbx6 & B4 & Alexa Fluor 594 \\
\hline
\end{tabular}


1 Preprocessing of the microscopy images was done using ImageJ. The sox 2 and tbx6 channels

2 were subtracted from each other to eliminate bleed through. Images were rotated to a consistent

3 orientation and a max intensity projection was created. Adaxial cells were identified in the DAPI

4 channel and manually removed from the image. The midline separating the embryo into left and

5 right halves was identified manually. Subsequent quantification was performed in Matlab. The

6 image was smoothed with a Gaussian filter, and the region of interest was thresholded using

7 Otsu's algorithm on both the $t b \times 16$ and $t b x 6$ channels. For wildtype, BMP, and FGF inhibited

8 embryos, average fluorescent intensity was measured along the $x$ axis of the image and

9 normalized to the maximum value. This was done separately for the left and right sides of the embryo. The PZ/PSM boundary was taken to be the first point with a value greater than $20 \%$ of the maximum tbx6 value. The anterior end of the PSM was defined as the last point greater than $85 \%$ of the maximum $t b x 6$ value. The scaled $P Z$ length was the $P Z$ length divided by the distance from the end of the tail to the anterior boundary of the PSM.

Wnt inhibited embryos had some modifications to the quantification. In bent embryos, the boundary separating the left and right halves was taken to be a line through the midpoint of the tailbud brachyury signal to the notochord and then following the notochord towards the head. For wild-type and Wnt inhibited embryos the outer perimeter of the embryo was traced manually. The curve was smoothed with a Savitzky-Golay filter and defined as the embryo's axis. Pixels in the ROI were mapped to their closest points on the perimeter using the distance2curve function from John D'Errico. The mean intensity along the axis was calculated using a sliding window. The same thresholds were used for the PZ and PSM as described previously. The boundaries for

22 these regions were taken to be a perpendicular dropped from the axis at the cutoff point. The scaled PZ area was the PZ area divided by the area of the PZ plus PSM. 


\section{References}

Schrödinger, E. (1944). What is Life? The Physcial Aspect of the Living Cell: Cambridge University Press.

Sulston, J. E., Schierenberg, E., White, J. G., \& Thomson, J. N. (1983). The embryonic cell lineage of the nematode Caenorhabditis elegans. Dev Biol, 100(1), 64-119. doi:10.1016/00121606(83)90201-4 Kimmel, C. B., Warga, R. M., \& Schilling, T. F. (1990). Origin and organization of the zebrafish fate map. Development, 108, 581-594.

Kanki, J. P., \& Ho, R. K. (1997). The development of the posterior body in zebrafish. Development, 124, 881-893.

Fearnhead, P. (2006). Exact and efficient Bayesian inference for multiple changepoint problems. Statistics and Computing, 16(2), 203-213. doi:10.1007/s11222-006-8450-8

Tzouanacou, E., Wegener, A., Wymeersch, F. J., Wilson, V., \& Nicolas, J. F. (2009). Redefining the progression of lineage segregations during mammalian embryogenesis by clonal analysis. Dev Cell, 17(3), 365-376. doi:10.1016/j.devcel.2009.08.002

Benazeraf, B., Francois, P., Baker, R. E., Denans, N., Little, C. D., \& Pourquie, O. (2010). A random cell motility gradient downstream of FGF controls elongation of an amniote embryo. Nature, 466(7303), 248-252. doi:nature09151 [pii]10.1038/nature09151 [doi]

Fior, R., Maxwell, A. A., Ma, T. P., Vezzaro, A., Moens, C. B., Amacher, S. L., . . Saude, L. (2012). The differentiation and movement of presomitic mesoderm progenitor cells are controlled by Mesogenin 1. Development, 139(24), 4656-4665. doi:10.1242/dev.078923

Madhulatha, T. S. (2012). An overview on clustering methods. IOSR Journal of Engineering, 2, 719-725. doi:

Martin, B. L., \& Kimelman, D. (2012). Canonical Wnt signaling dynamically controls multiple stem cell fate decisions during vertebrate body formation. Dev Cell, 22(1), 223-232.

doi:10.1016/j.devcel.2011.11.001

Yabe, T., \& Takada, S. (2012). Mesogenin causes embryonic mesoderm progenitors to differentiate during development of zebrafish tail somites. Dev Biol, 370(2), 213-222. doi:10.1016/j.ydbio.2012.07.029 Dray, N., Lawton, A. K., Nandi, A., Jülich, D., Emonet, T., \& Holley, S. A. (2013). Cell-Fibronectin interactions propel vertebrate trunk elongation via tissue mechanics. Curr Biol, 23, 1335-1341. Lawton, A. K., Nandi, A., Stulberg, M. J., Dray, N., Sneddon, M. W., Pontius, W., . . Holley, S. A. (2013). Regulated tissue fluidity steers zebrafish body elongation. Development, 140, 573-582. Wilson, C., High, S., McCluskey, B., Amores, A., Yan, Y., Titus, T., . . Postlethwait, J. (2014). Wild sex in zebrafish: loss of the natural sex determinant in domesticated strains. Genetics, 198(3). doi:10.1534/genetics.114.169284

Manning, A. J., \& Kimelman, D. (2015). Tbx16 and Msgn1 are required to establish directional cell migration of zebrafish mesodermal progenitors. Dev Biol, 406, 172-185.

doi:10.1016/j.ydbio.2015.09.001

Moore, C. C. (2015). Ergodic theorem, ergodic theory, and statistical mechanics. Proceedings of the National Academy of Sciences, 112(7), 1907-1911. doi:10.1073/pnas.1421798112

Satija, R., Farrell, J. A., Gennert, D., Schier, A. F., \& Regev, A. (2015). Spatial reconstruction of single-cell gene expression data. Nature Biotechnology, 33(5), 495-502. doi:10.1038/nbt.3192 
Steventon, B., Duarte, F., Lagadec, R., Mazan, S., Nicolas, J. F., \& Hirsinger, E. (2016). Species tailoured contribution of volumetric growth and tissue convergence to posterior body elongation in vertebrates. Development, 143, 1732-1741. doi:10.1242/dev.126375 Das, D., Chatti, V., Emonet, T., \& Holley, S. A. (2017). Patterned disordered cell motion ensures vertebral column symmetry. Dev Cell, 42, 170-180. doi:10.1016/j.devcel.2017.06.020 Gouti, M., Delile, J., Stamataki, D., Wymeersch, F. J., Huang, Y., Kleinjung, J., . . Briscoe, J. (2017). A Gene Regulatory Network Balances Neural and Mesoderm Specification during Vertebrate Trunk Development. Dev Cell, 41(3), 243-261. doi:10.1016/j.devcel.2017.04.002 Thomas, P. (2017). Making sense of snapshot data: ergodic principle for clonal cell populations. $J$ $R$ Soc Interface, 14(136). doi:10.1098/rsif.2017.0467

Choi, H. M. T., Schwarzkopf, M., Fornace, M. E., Acharya, A., Artavanis, G., Stegmaier, J., . . . Pierce, N. A. (2018). Third-generation in situ hybridization chain reaction: multiplexed, quantitative, sensitive, versatile, robust. Development, 145(12), dev165753.

doi:10.1242/dev.165753

Farrell, J. A., Wang, Y., Riesenfeld, S. J., Shekhar, K., Regev, A., \& Schier, A. F. (2018). Single-cell reconstruction of developmental trajectories during zebrafish embryogenesis. Science, 360(6392), eaar3131. doi:10.1126/science.aar3131

Mongera, A., Rowghanian, P., Gustafson, H. J., Shelton, E., Kealhofer, D. A., Carn, E. K., . . Campas, O. (2018). A fluid-to-solid jamming transition underlies vertebrate body axis elongation. Nature, 561(7723), 401-405. doi:10.1038/s41586-018-0479-2

Simon, C. S., Hadjantonakis, A. K., \& Schröter, C. (2018). Making lineage decisions with biological noise: Lessons from the early mouse embryo. Wiley Interdiscip Rev Dev Biol, 7(4), e319. doi:10.1002/wdev.319 Wagner, D. E., Weinreb, C., Collins, Z. M., Briggs, J. A., Megason, S. G., \& Klein, A. M. (2018). Single-cell mapping of gene expression landscapes and lineage in the zebrafish embryo. Science, 360(6392), 981-987. doi:10.1126/science.aar4362

Das, D., Jülich, D., Schwendinger-Schreck, J., Guillon, E., Lawton, A. K., Dray, N., . . Holley, S. A. (2019). Organization of embryonic morphogenesis via mechanical information. Dev Cell, 49, 829839.

Hafemeister, C., \& Satija, R. (2019). Normalization and variance stabilization of single-cell RNAseq data using regularized negative binomial regression. Genome biology, 20(1). doi:10.1186/s13059-019-1874-1

Bergen, V., Lange, M., Peidli, S., Wolf, F. A., \& Theis, F. J. (2020). Generalizing RNA velocity to transient cell states through dynamical modeling. Nature Biotechnology, 38(12), 1408-1414. doi:10.1038/s41587-020-0591-3 
A

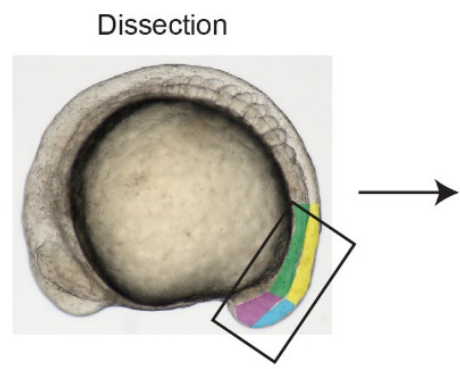

B

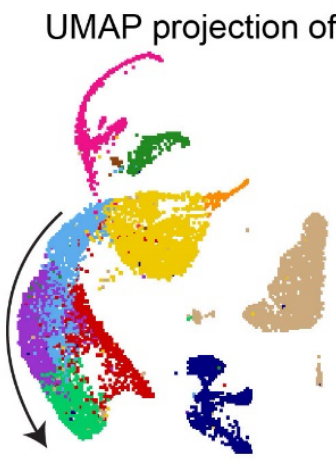

Single Cell RNAseq

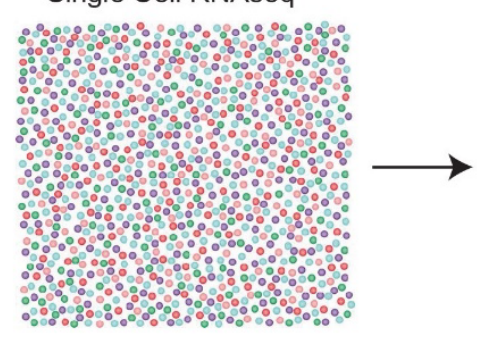

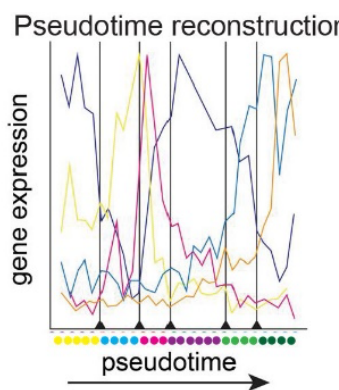

C Wild type pseudotime sequence

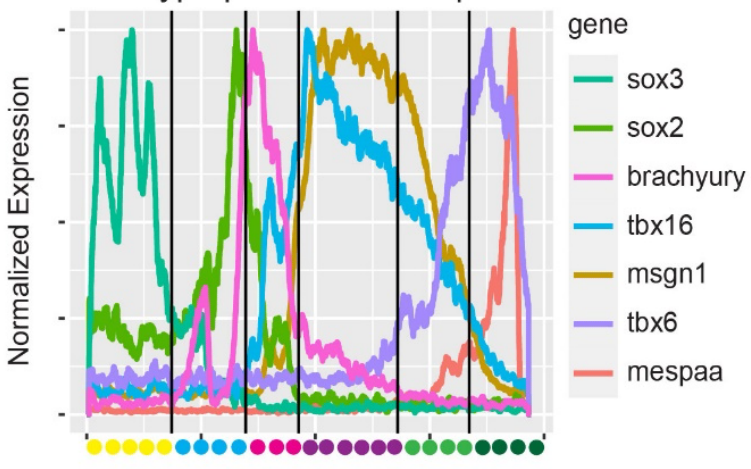

Pseudotime

- Posterior neural tube

Dorsalmedial zone

- Progenitor zone

- PSM

- Adaxial cells

- Differentiated neurons

- Lateral plate mesoderm

- Epidermis

- Notochord

- Pronephric progenitors

- Kupffer's vesicle

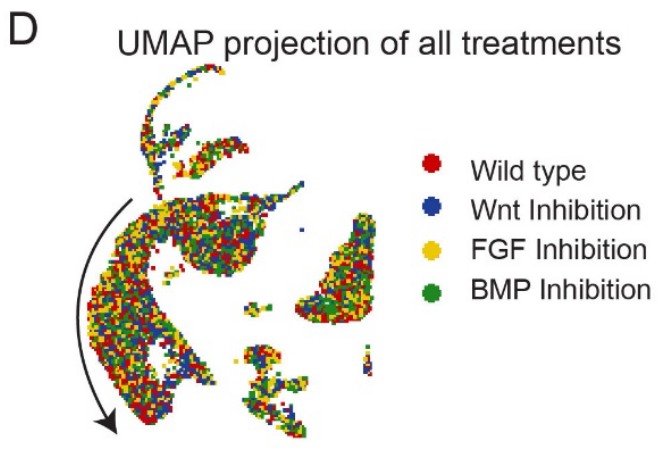

Fig. 1. Gene expression cell states in the zebrafish tailbud. (A) Schematic of the experimental approach. Tailbuds were dissected and pooled, scRNAseq profiles were generated, and a onedimensional pseudotime was created and segmented into gene expression cell states. (B) UMAP projection of scRNAseq data colored by cell type. Arrow marks the path of pseudotime in C. (C) Expression of selected markers over pseudotime. Vertical lines are transition points between cell states as defined by a Bayesian algorithm that minimizes within state statistical error. Note that the segment colors along the pseudotime axis correspond to the colors along the developmental trajectory (arrow) in B but with the Progenitor Zone and PSM being further subdivided into two similarly colored segments in C. (D) UMAP projection of scRNAseq data colored by experimental treatment. (E) Quantification of the differences in the proportion of cells that are in a given cell state in each replicate of each experimental condition. See also Figs. S1, S2 and Table S1. 
A Cell state mapping by in situ hybridization

$$
i
$$
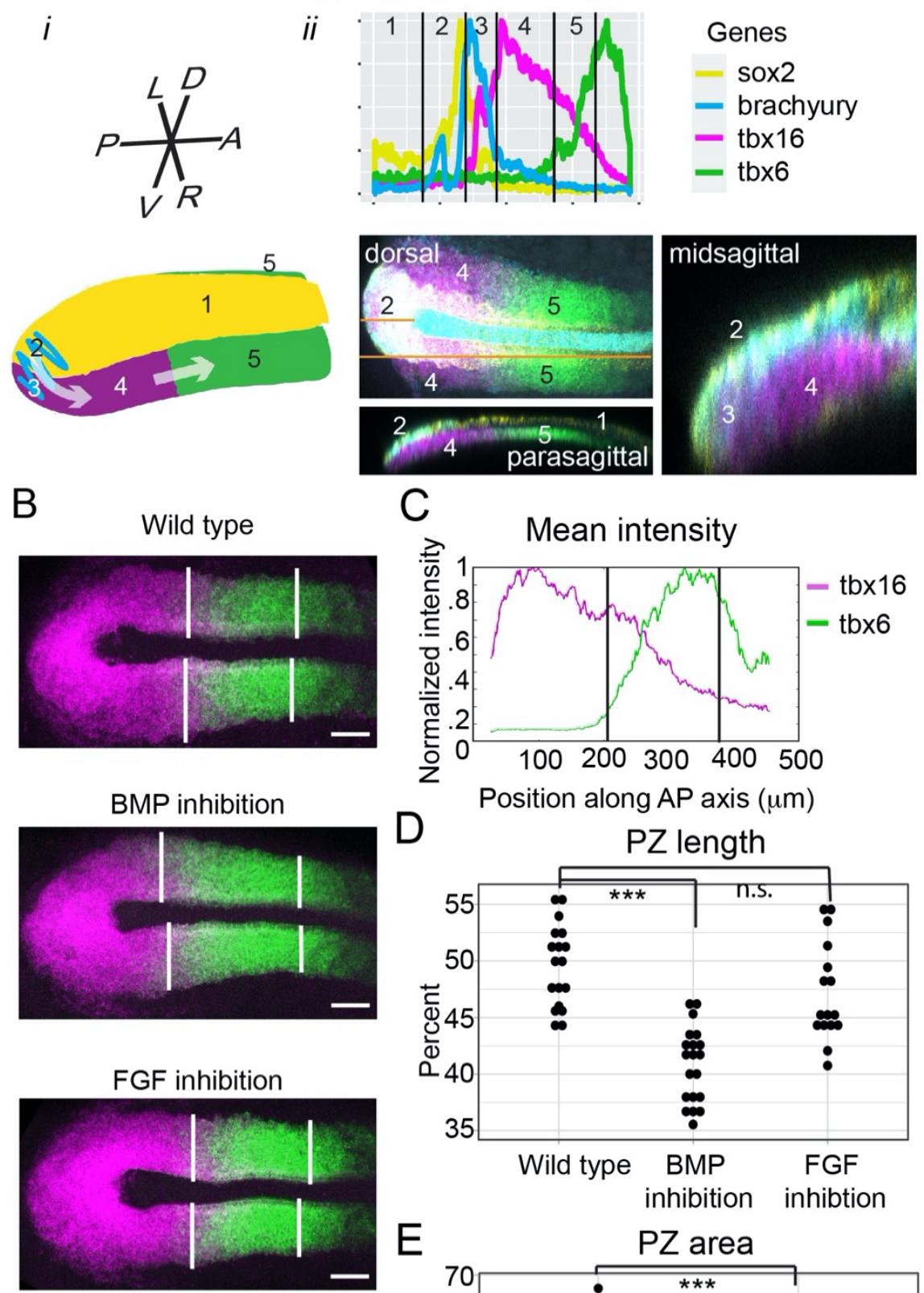

Wnt inhibtion

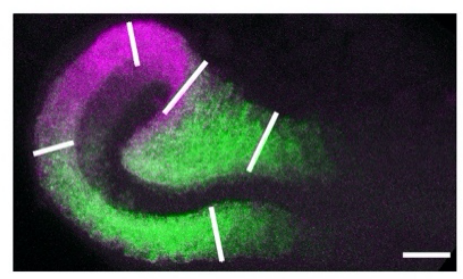

\section{C}

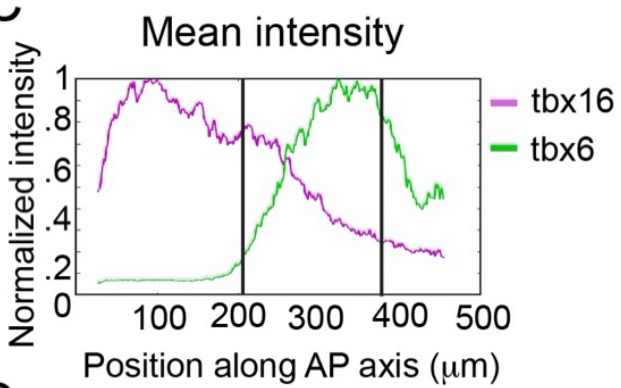

D

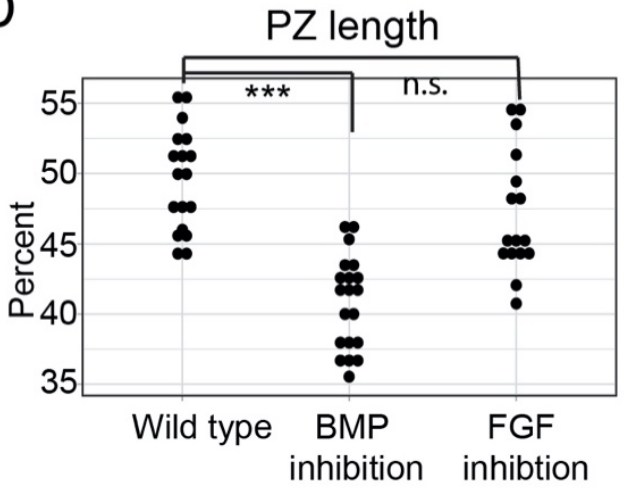

$\mathrm{E}$

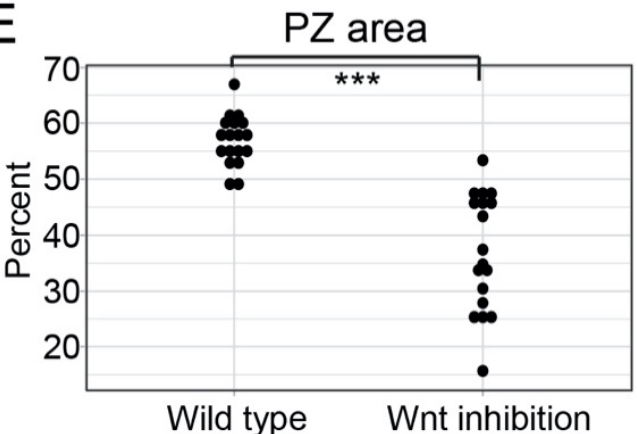

Fig. 2. scRNAseq gene expression states map to the tailbud. (A) (i) A schematic showing the developmental trajectory of the paraxial mesoderm in the tailbud. All panels show the expression of sox2 (yellow), brachyury (cyan), tbx16 (magenta), and tbx6 (green). (ii) Fluorescent in situ hybridization maps the transcriptional states (numbered) defined by scRNAseq onto the tailbud. In the dorsal view, the orange lines mark locations of the midsagittal (short line) and parasagittal (long line) slices. (B) Expression of tbx16 (magenta) and tbx6 (green). Vertical lines mark the transition from PZ to PSM and PSM to anterior PSM. Scale bar is 50 microns. (C) Plot of signal intensity in a representative wild-type embryo along the anterior-posterior axis. Vertical bars are cutoffs for the PZ and PSM of $20 \%$ and $85 \%$ of maximum tbx 6 expression, respectively. (D) PZ length normalized to total length of PZ and PSM in wild-type, BMP and FGF inhibited embryos. (E) PZ area normalized to total area in wild-type and Wnt inhibited embryos. ${ }^{* * *}$ is $p<.001$. See also Fig. S3. 

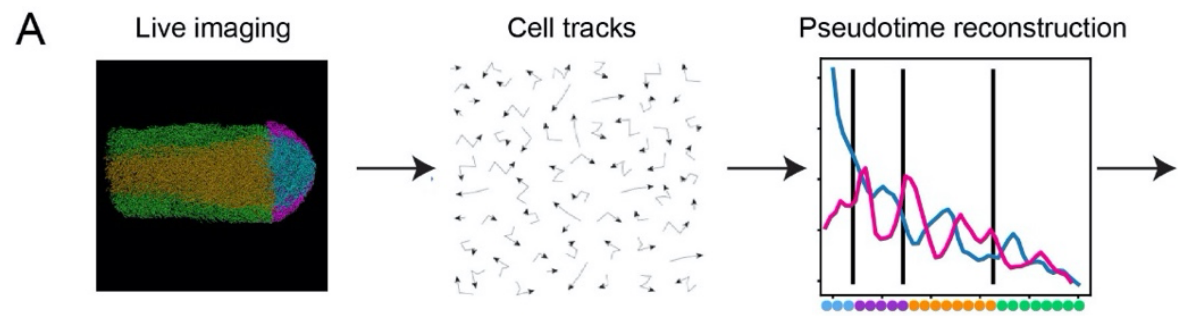

B

C
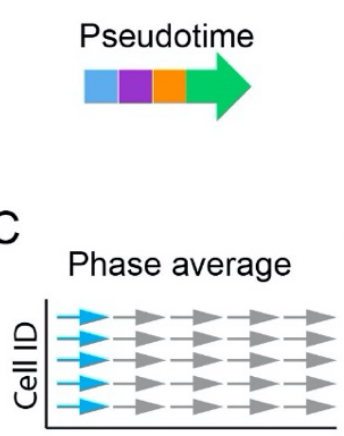

Time

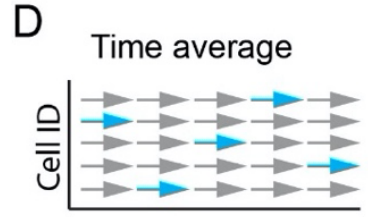

Time

$\mathrm{E}$

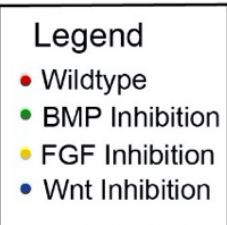

Pseudotime segmentation without position
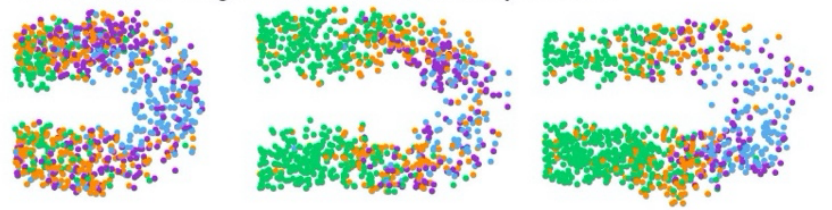

Pseudotime segmentation with position. Cells plotted from $t=1$
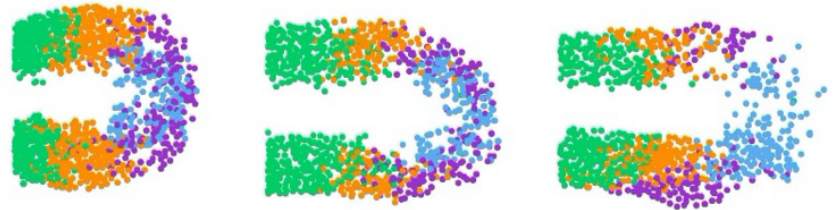

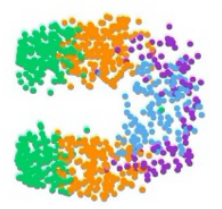

PZ dynamics

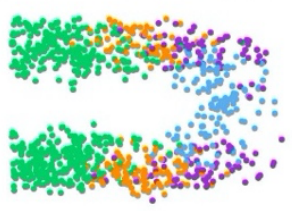

$\mathrm{F}$ Movie S1.
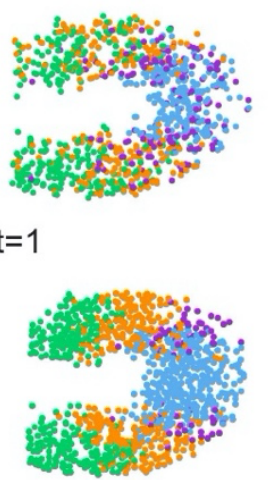

Pseudotime segmentation with position. Cells plotted from all timepoints

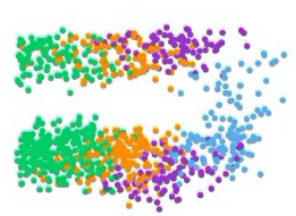

Posterior PSM dynamics.

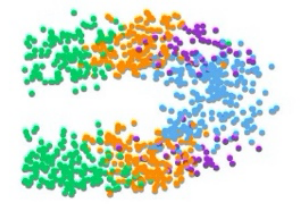

Abundance

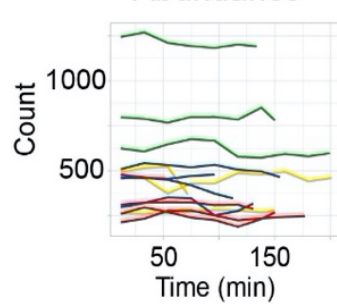

Fig. 3. The pattern of cell motion states is ergodic. (A) Conceptual approach to identifying cell motion states. Tailbuds are imaged in 4D and cells are tracked, position is removed from cell tracks and data from multiple embryos are pooled, cell motion statistics used to construct and segment pseudotime, and cell states mapped back onto the embryo using the original cell position. (B, C and D) Cell motion state patterns of four wild-type embryos generated using three variations of this method. Each plot is a dorsal view with anterior orientated to the left. Each point is one cell, colored by migration state. (B) Plot of the segmentation of four embryos using a set of five cell motion parameters and data pooled from all embryos. ( $C$ and $D$ ) Cell state patterns generated individually for each embryo using cell position and cell track displacement for pseudotime estimation. For plotting, 1000 cells were chosen at random from each embryo from either the first timepoint $(C)$ or from all timepoints $(D)$. Note the similar patterns for each embryo, i.e. the vertically aligned cell state plots in $C$ and $D$. (E and F) The PZ (E) and posterior PSM (F) cell counts over time and plots of cell influx vs outflux for each state. See also Fig. S4 and S5 and 
A RNA velocity in wildtype

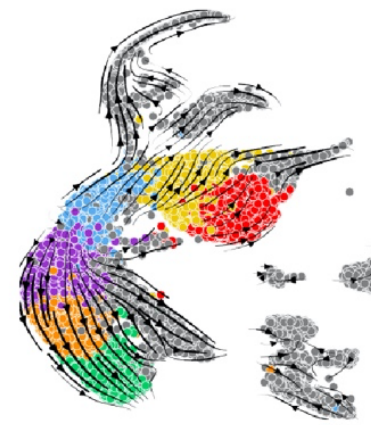

B

$$
\begin{aligned}
& \text { Legend } \\
& \text { - Wild type } \\
& \text { - BMP Inhibition } \\
& \text { - FGF Inhibition } \\
& \text { - Wnt Inhibition }
\end{aligned}
$$

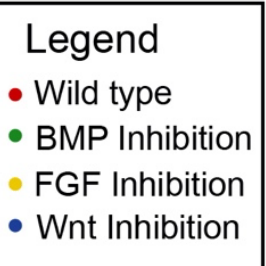

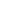

C tbx16 in situ hybridization

Neuronal cells

NMP

Mesodermal Progenitors

$P Z$

Posterior PSM

Anterior PSM

Other

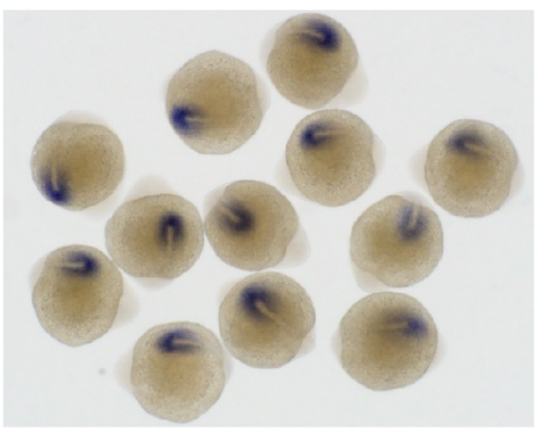

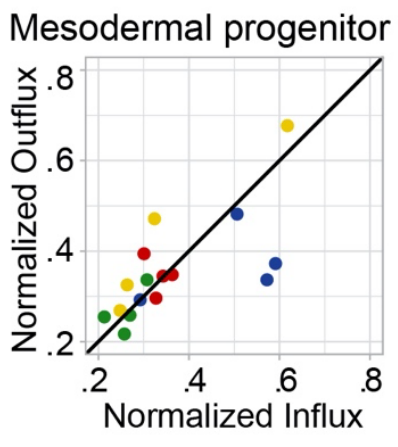
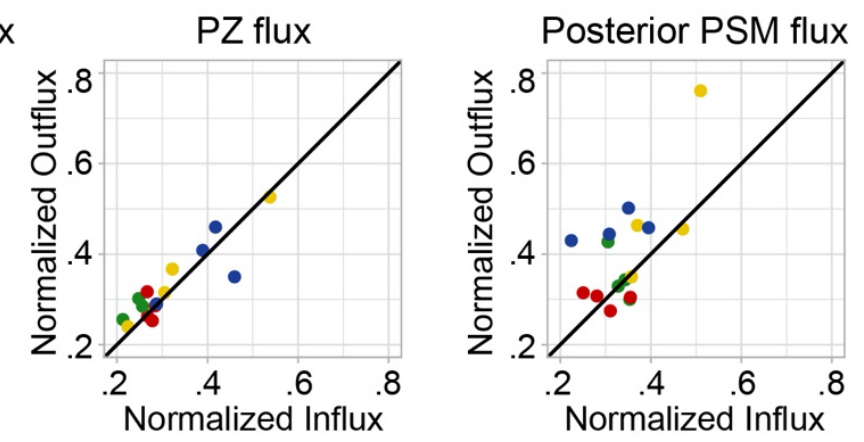

Fig. 4. Flux analysis of gene expression cell states is consistent with ergodicity. (A) RNA velocity UMAP plot of tailbud gene expression states. (B) Influx vs outflux plots estimated by RNA velocity. Note that the influx and outflux are balanced in wild-type embryos, but cell signaling perturbation can alter this balance. (C) In situ hybridization for tbx16 among sibling embryos illustrates the reproducibility of embryonic development. See also Fig. S6 and S7. 
A

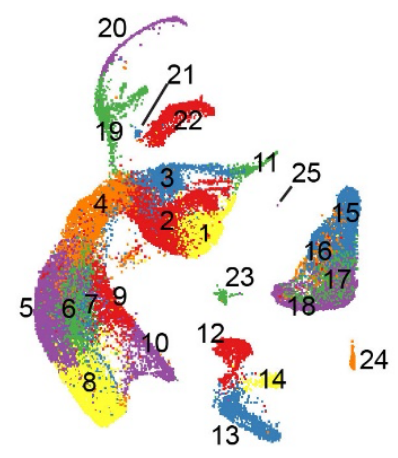

B
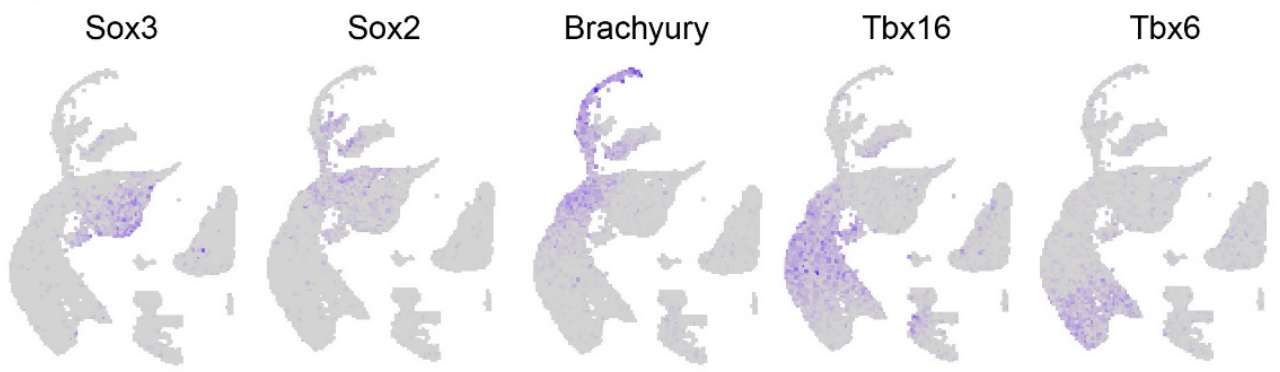

13 Lateral plate mesoderm (Imo2+)

2 Posterior neural tube

- 3 Dorsal neural tube

- 4 Neuromesodermal progenitor

- $5 \mathrm{PZ}$

- $6 \mathrm{PZ}$

- $7 \mathrm{PZ}$

8 Presomitic Mesoderm

- 9 Posterior adaxial cells

- 10 Anterior adaxial cells

11 Differentiated Neurons

- 12 Lateral plate mesoderm (hand2+)
14 Lateral plate mesoderm (pax2a+)

- 15 Epidermis

16 Epidermis

17 Epidermis

- 18 Epidermis

- 19 Posterior notochord

- 20 Anterior notochord

- 21 Kupffer's vesicle

- 22 Intermediate mesoderm

- 23 EVL

- 24 Mucus producing cells

- 25 Polster

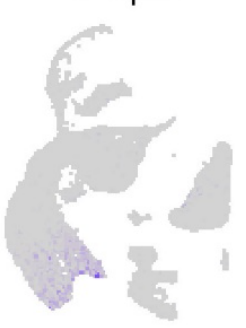

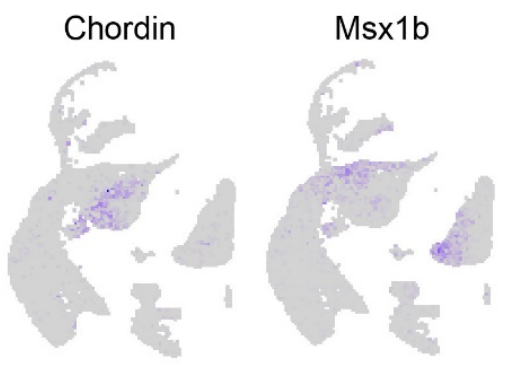
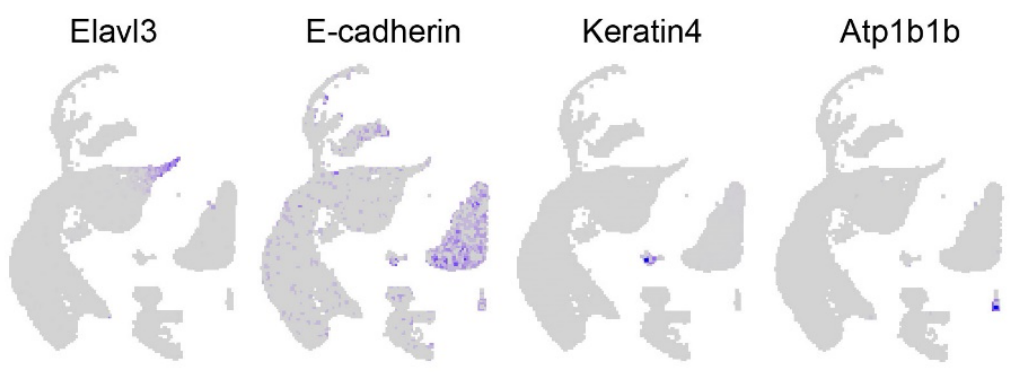

Myod1
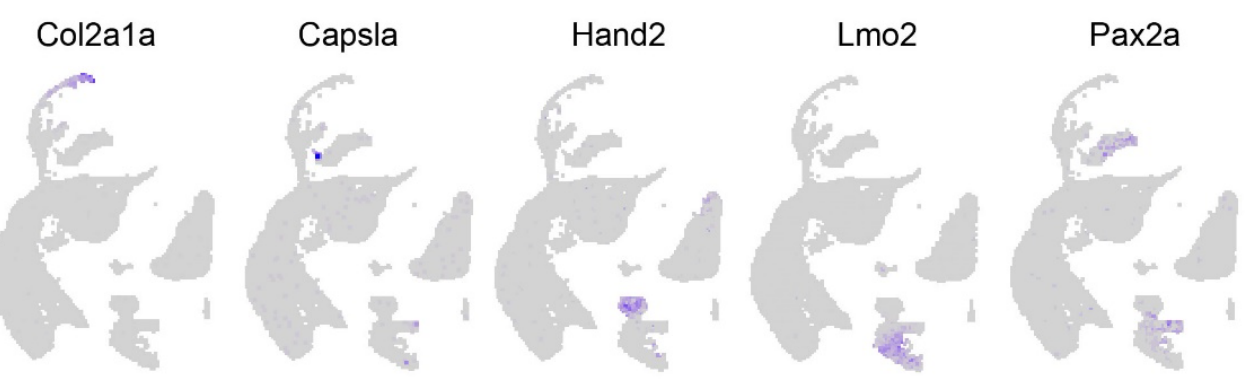

\section{Expression}

0

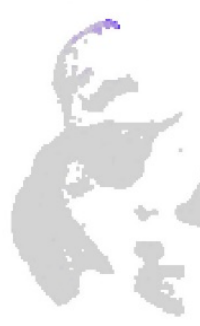

2 Figure S1. Gene expression states from scRNAseq. Related to Figure 1. (A) UMAP projection of cell clusters defined by Louvain clustering using Seurat. Clusters were manually annotated using marker genes. (B) UMAP projection of selected marker genes used to identify cell types. 


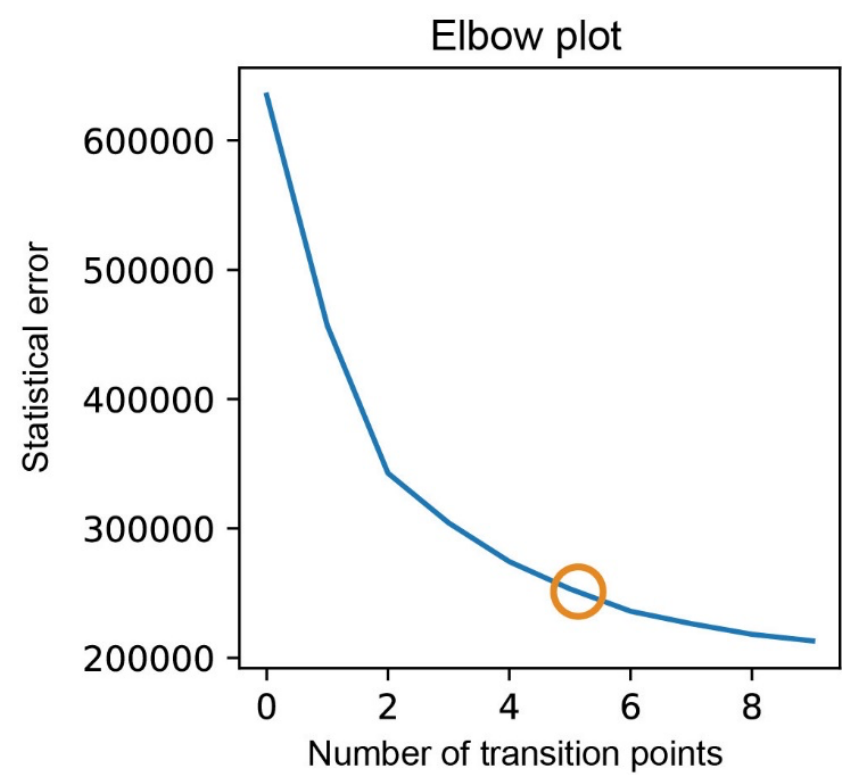

Figure S2. Segmentation of gene expression pseudotime. Related to Figure 1. An elbow plot used to select the number of transition points in the scRNAseq pseudotime. Circle marks the 4 chosen number of transitions. 

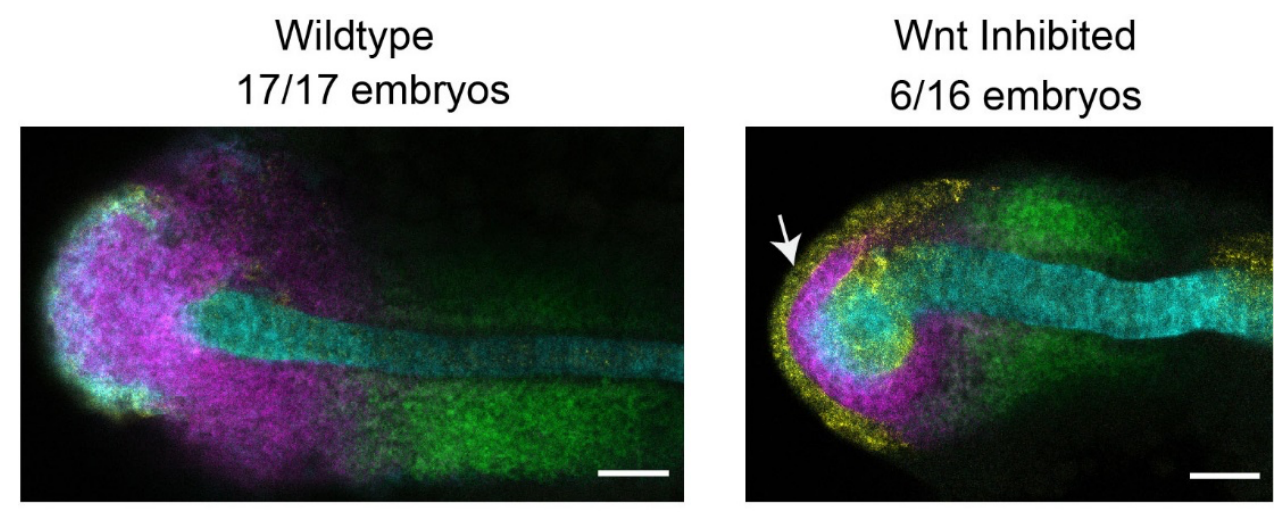

Figure S3. Wnt inhibited embryos have excessive neural tissue. Related to Figure 2. Fivemicron thick projections through the ventral tailbud of a multicolor fluorescent in situ hybridization. Arrow points to inappropriately located sox 2 single positive neural tissue. Scale bar $=50$ microns 
A
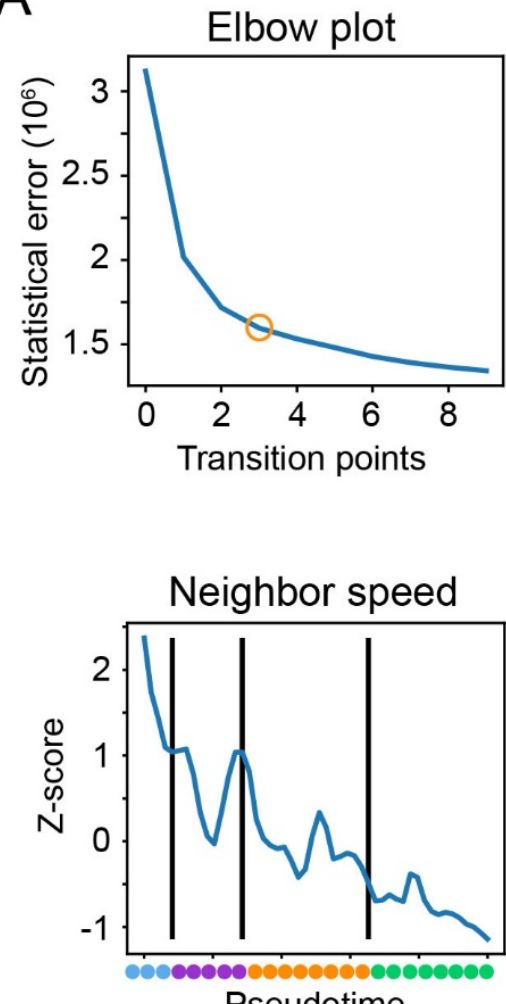
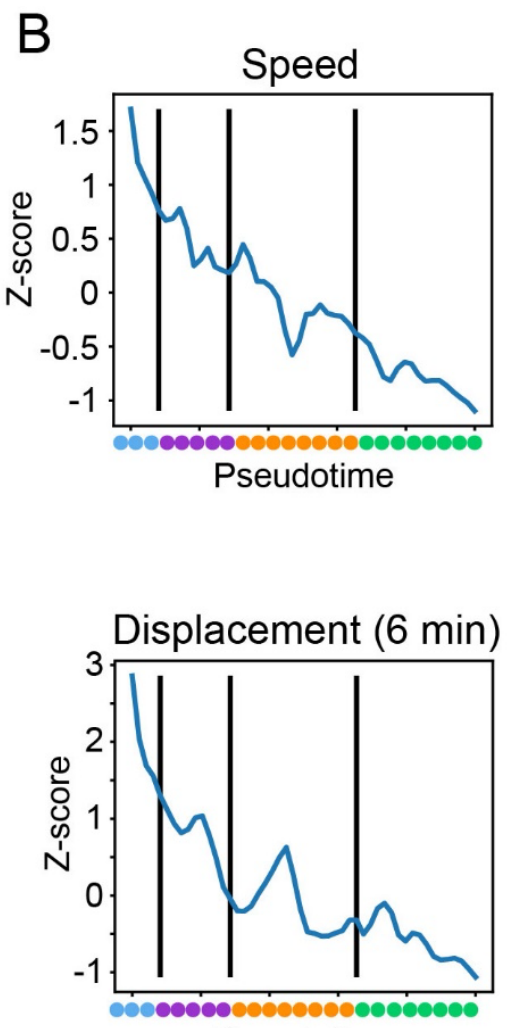

Pseudotime

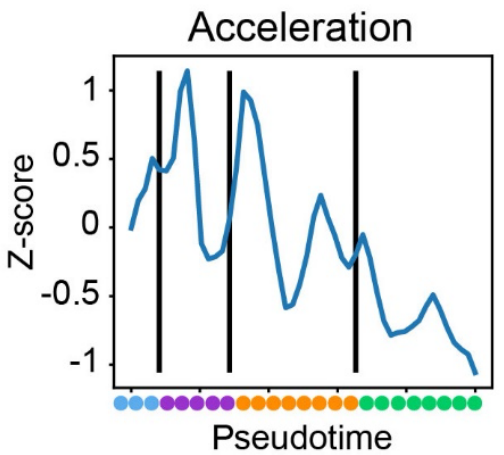

Displacement (15 min)

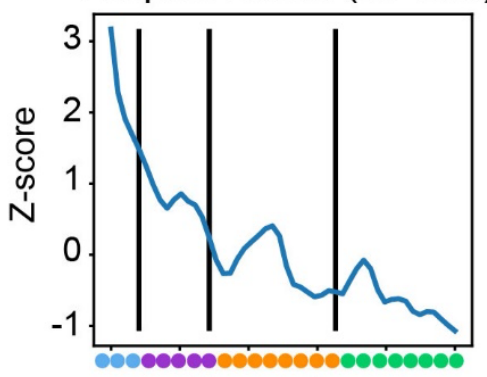

Pseudotime

Figure S4. Cell motion pseudotime segmentation. Related to Figure 3. (A) An elbow plot used to select the number of transition points in the cell motion pseudotime. Circle marks the chosen number of transitions. (B) Cell motion statistics used to segment the embryos in Fig 3B plotted over pseudotime. Neighbor speed represents the average cell speed withing a $20 \mu \mathrm{m}$ radius of each cell. Displacement statistics were analyzed over two time intervals. Vertical lines mark transition points. The segment colors along the pseudotime axis correspond to the cell motion state colors in Fig. 2 and S5. 


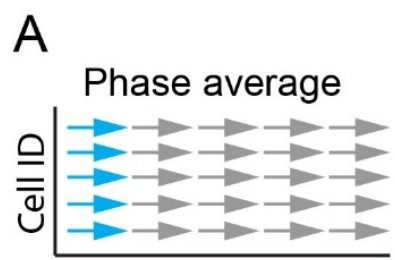

Time

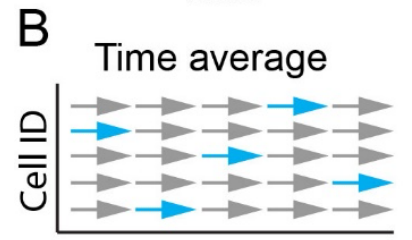

Time

C

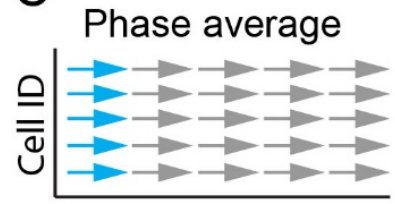

Time

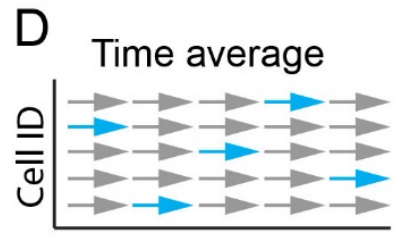

Time

E

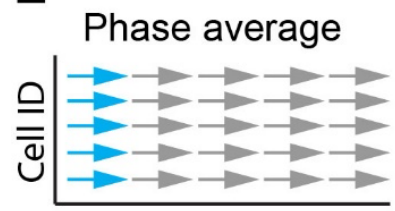

Time

$\mathrm{F}$

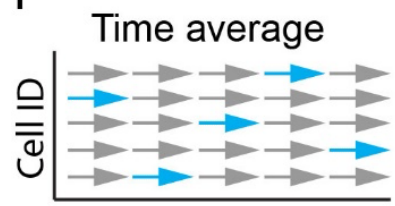

Time
BMP inhibition
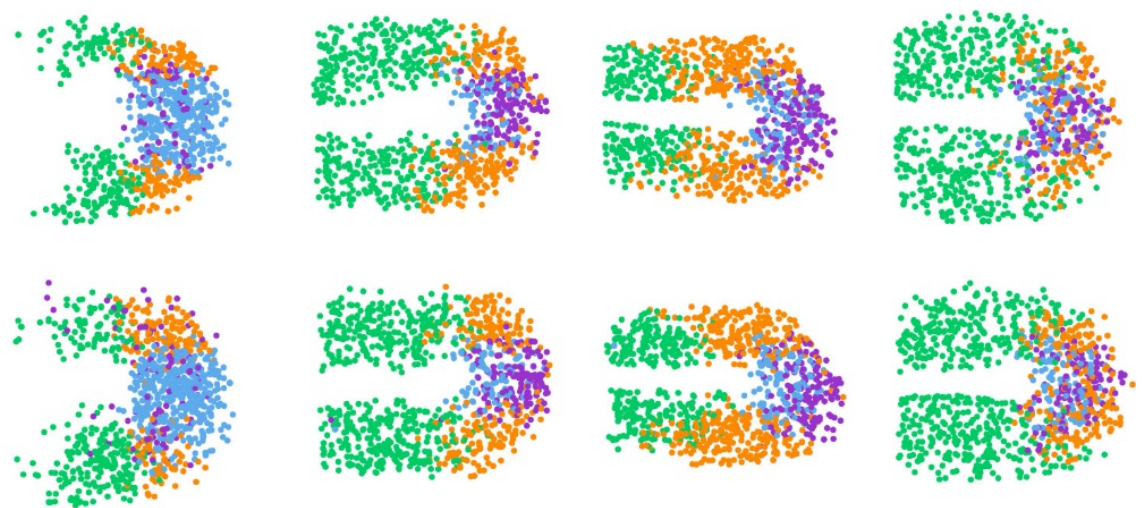

FGF inhibition
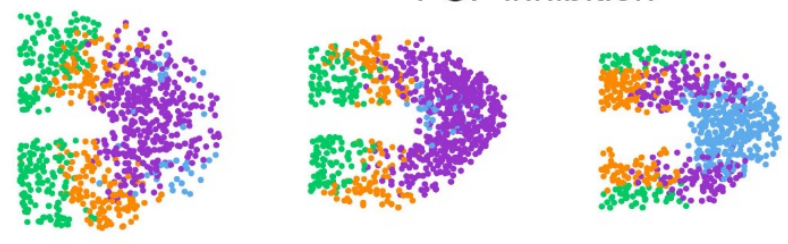

Pseudotime
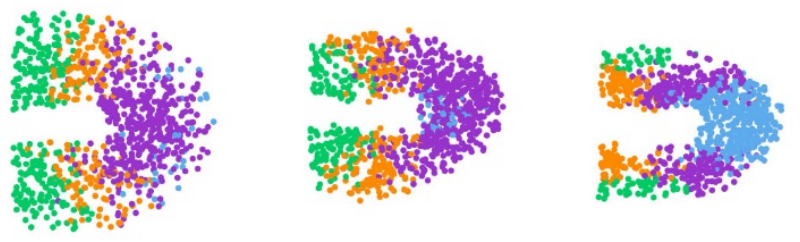

Wnt inhibition
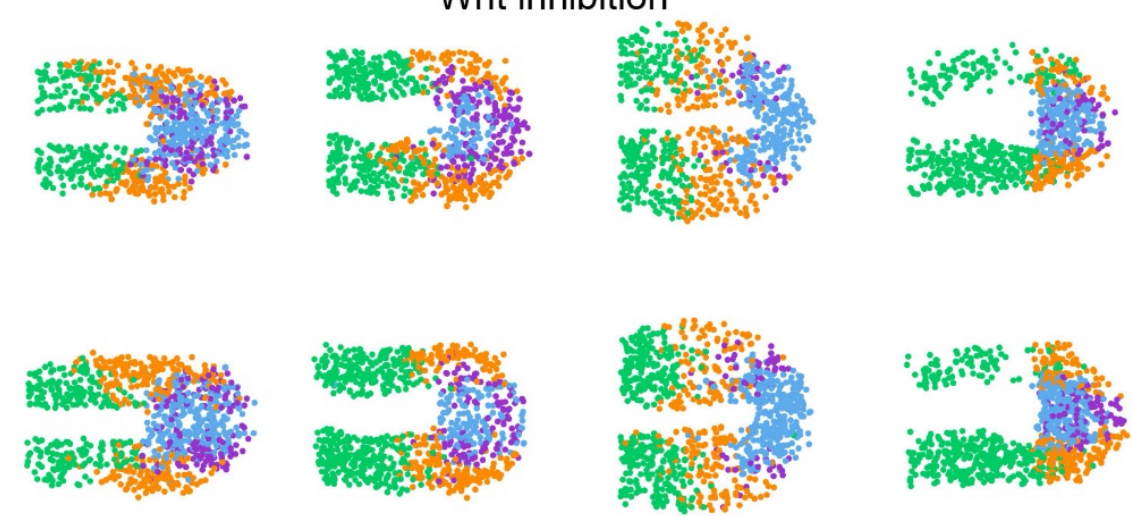

Figure S5. The pattern of cell migration states remains ergodic in embryos subject to signaling perturbations. Related to Figure 3. Data from embryos subject to signaling perturbations, processed and plotted identically to the wildtype embryos in Fig 3C. (A) The phase average plots for four embryos with reduced Bmp signaling. (B) Time average plots for the same four embryos with reduced Bmp signaling. (C) The phase average plots for three embryos with reduced FGF signaling. (D) Time average plots for the same three embryos with reduced FGF signaling. (E) The phase average plots for four embryos with reduced Wnt signaling. (F) Time average plots for the same four embryos with reduced Wnt signaling. 

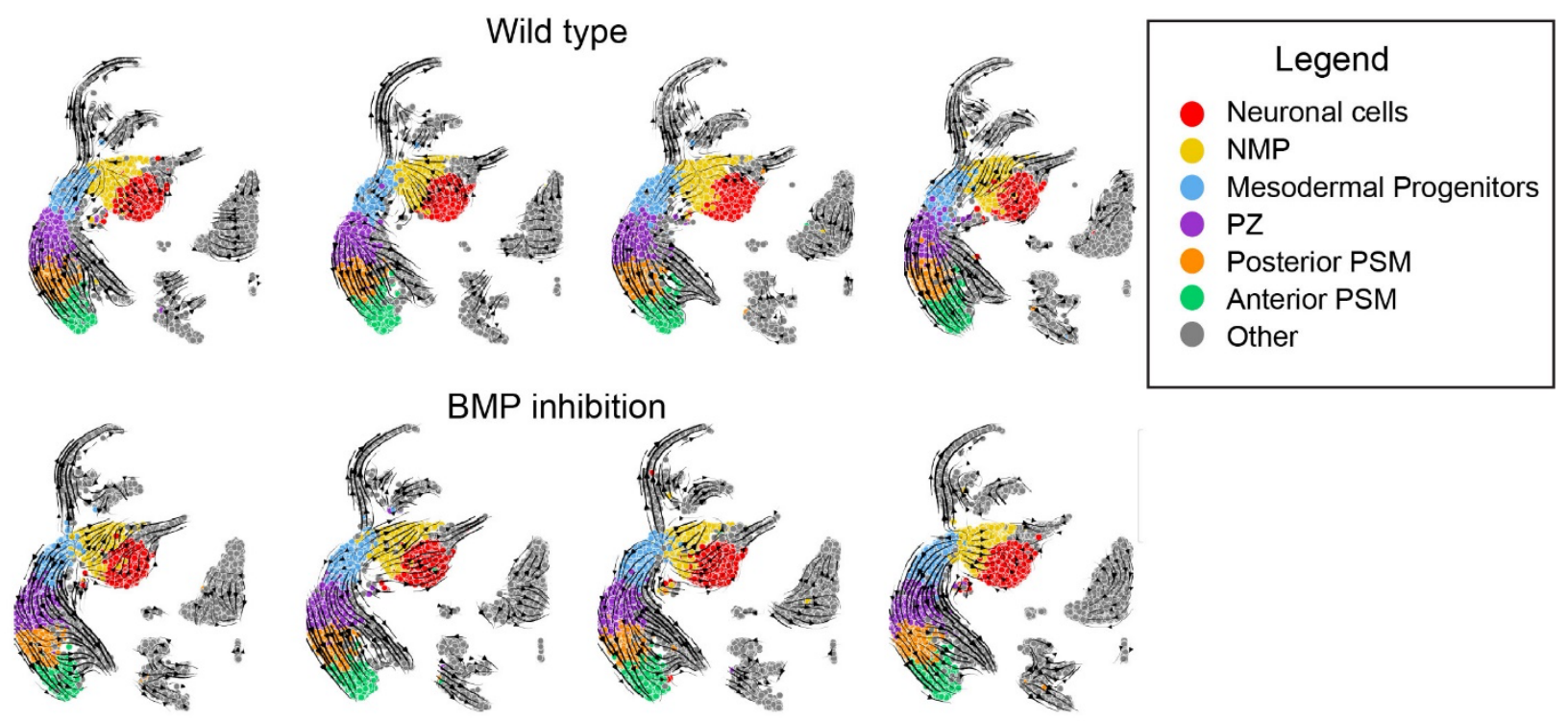

FGF inhibition
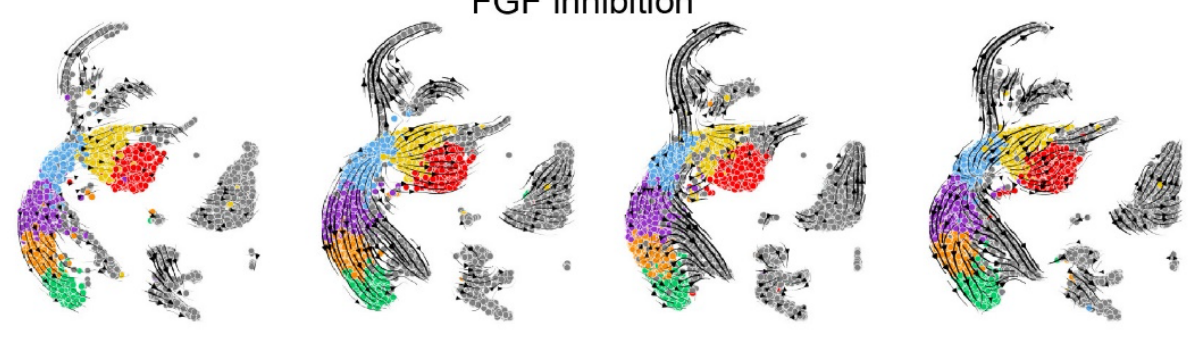

Wnt inhibition
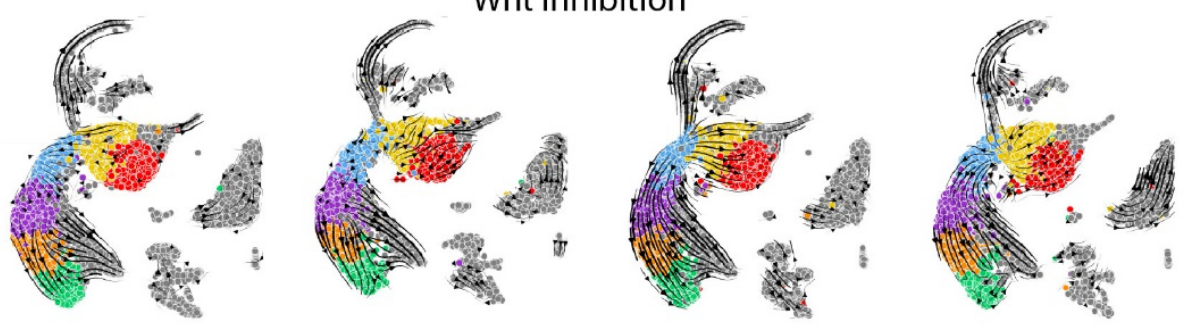

Figure S6. RNA velocity plots of all scRNAseq replicates. Related to Figure 4. Plots for all replicates were used to calculate cell state flux in Fig 4B. 

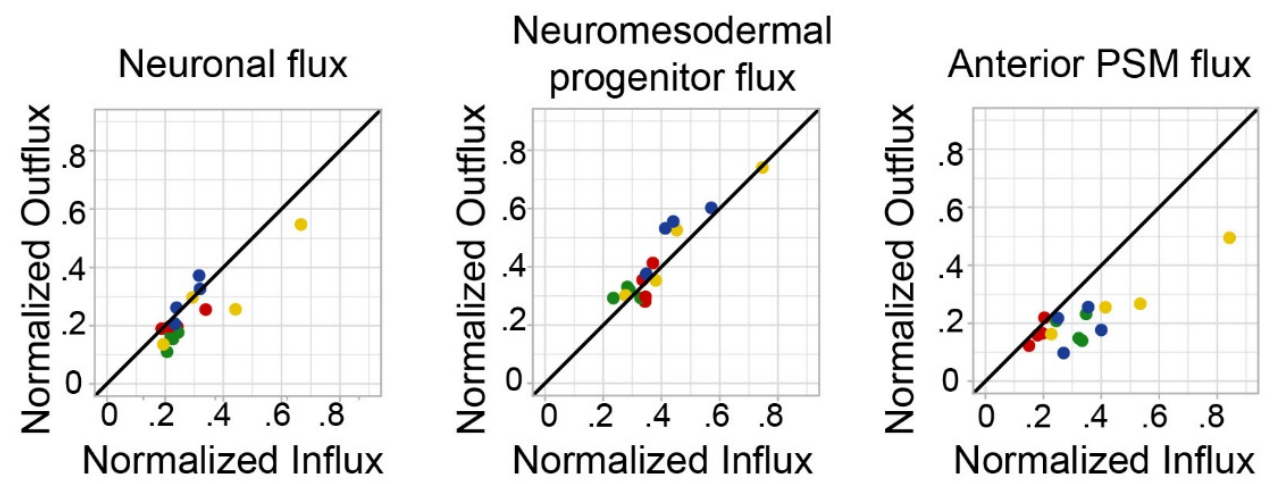

2 Figure S7. Cell flux through tailbud cell states derived from RNA velocity. Related to

3 Figure 4. Influx is greater than outflux for the anterior PSM and neuronal states. However, this

4 may be an artifact of the absence of further differentiated tissues in the dataset. 


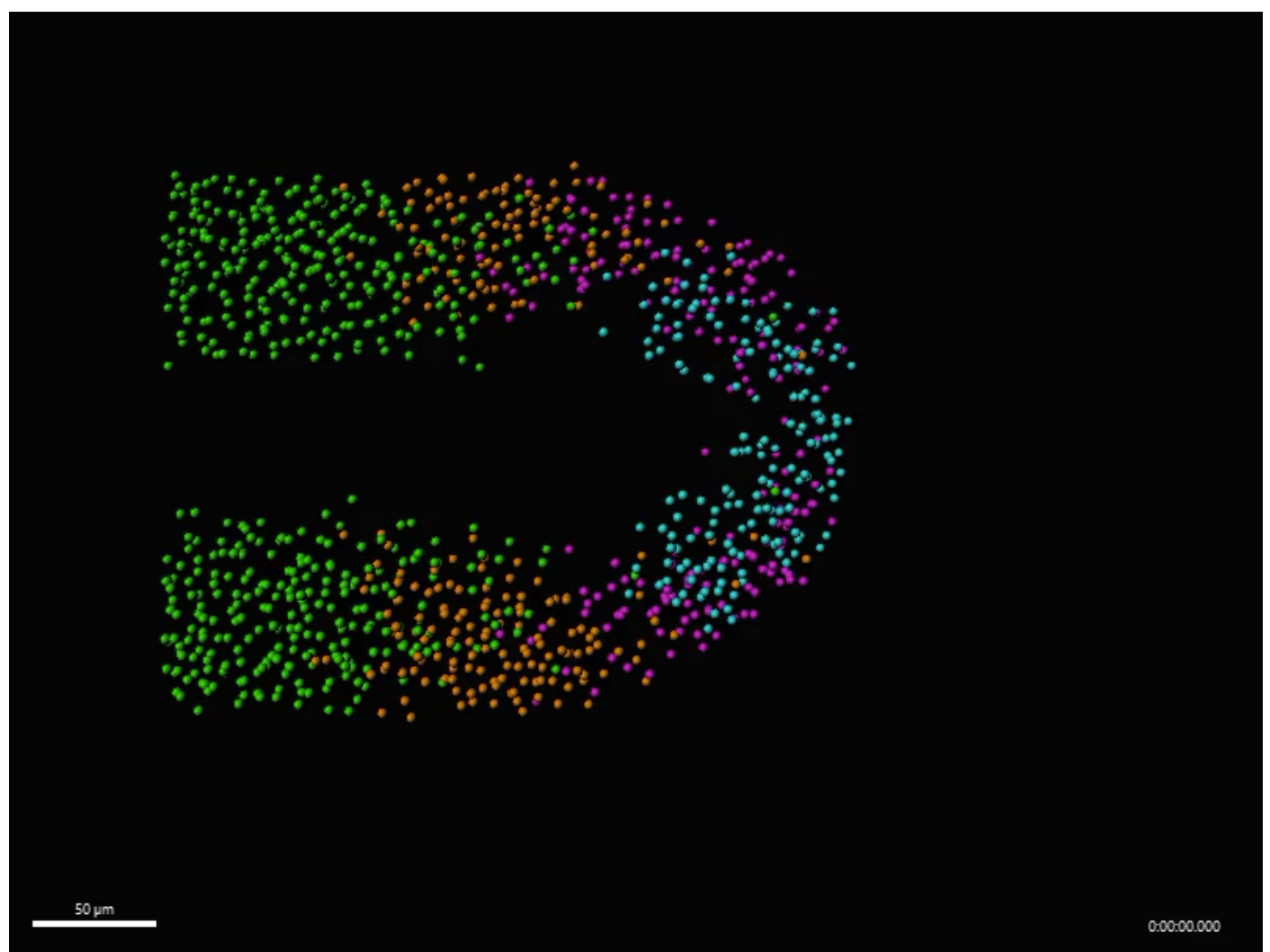

2 Movie S1. Ergodic pattern of cell state transitions. Related to Figure 3. A timelapse was 3 generated using the phase average for each timepoint in the longest wild-type dataset. The 4 states were segmented using position and displacement. 


\begin{tabular}{|l|r|r|r|}
\hline Samples & Max gene number & Minimum gene number & $\begin{array}{l}\text { Max mitochondrial } \\
\text { percentage }\end{array}$ \\
\hline WT1 & 2800 & 1200 & 5 \\
\hline WT2 & 2000 & 800 & 5 \\
\hline WT3 & 2500 & 800 & 5 \\
\hline WT4 & 2500 & 800 & 7 \\
\hline BMP1 & 4000 & 1000 & 7 \\
\hline BMP2 & 3000 & 1000 & 7 \\
\hline BMP3 & 4000 & 1000 & 10 \\
\hline BMP4 & 4000 & 1000 & 7 \\
\hline FGF1 & 2000 & 800 & 7 \\
\hline FGF2 & 2500 & 800 & 7 \\
\hline FGF3 & 3000 & 800 & 3 \\
\hline FGF4 & 4000 & 1500 & 3 \\
\hline WNT1 & 2500 & 500 & 3 \\
\hline WNT2 & 2500 & 500 & 7 \\
\hline WNT3 & 3000 & 500 & 7 \\
\hline WNT4 & 3750 & & 7 \\
\hline TableS1 F & & & 7 \\
\hline
\end{tabular}

2 TableS1. Filtering criterions for the cells of scRNA-seq in each sample. Related to Figure

1. 